\title{
Complement levels and risk of organ involvement in patients with systemic lupus erythematosus
}

Ignacio Javier Gandino, Marina Scolnik, Emmanuel Bertiller, Valeria Scaglioni, Luis Jose Catoggio, Enrique Roberto Soriano

To cite: Gandino IJ, Scolnik M, Bertiller $\mathrm{E}$, et al. Complement levels and risk of organ involvement in patients with systemic lupus erythematosus. Lupus Science \& Medicine 2017;4. doi:10.1136/ lupus-2017-000209

Received 19 January 2017 Revised 3 May 2017 Accepted 7 May 2017

\section{CrossMark}

Hospital Italiano de Buenos Aires, Rheumatology Section, Medical Services. Instituto Universitario Hospital Italiano de Buenos Aires, and Fundacion PM Catoggio., Buenos Aires, Argentina

Correspondence to Professor Enrique Roberto Soriano; enrique.soriano@ hospitalitaliano.org.ar

\section{ABSTRACT}

Objective Complement plays a major role in SLE. Complement participation has been linked to disease activity and damage. Our objective was to estimate the association of complement behaviour with clinical manifestations, visceral injury and mortality in patients with SLE.

Methods Complement determinations (C3 and C4 levels) were analysed in patients with SLE (fulfilling American College of Rheumatology (ACR) or Systemic Lupus International Collaborating Clinics (SLICC)criteria) seen at a university hospital between 2000 and 2013. Patients were grouped in those with permanent $\mathrm{C} 3$ and/or C4 low values (low complement group), those with C3 and C4 constant normal values (normal complement group) and those with fluctuant values (periods of normal and periods of low values: fluctuant group). Clinical characteristics and mortality were analysed and compared between groups.

Results 270 patients with SLE were included (242 females, $89.6 \%$ ), mean age at diagnosis was 34.2 years (SD 15.8). 75 patients had fluctuant levels of complement, 79 patients had persistent low complement levels and 116 had normal complement levels. Lupus glomerulonephritis was more frequent in patients with fluctuant levels ( $75 \%, 56 \%$ and $49 \%$, respectively, $p=0002)$. The normal complement group had less frequency of haematological involvement and anti-double stranded DNA (dsDNA) antibodies. At the end of the follow-up, $53 \%$ of the patients had damage (SLICC/ACR $\geq 1$ ). In a Cox proportional hazard model age at diagnosis, neurological impairment, thrombocytopaenia and corticosteroids were associated with more damage, while hydroxychloroquine was a protective factor. There were no differences between complements groups on accumulated damage. Ten-year survival rate was $93 \%, 93.5 \%$ and $92 \%$ for the normal complement group, the persistently low group and the fluctuant group, respectively.

Conclusions Patients with constant normal complement had lower prevalence of haematological involvement and anti-dsDNA, while patients with fluctuant complement had higher renal impairment. Neither the persistent low complement nor the fluctuant complement groups had increased mortality and/or visceral damage.

\section{INTRODUCTION}

SLE is a chronic autoimmune disease characterised by a large number of autoantibodies and fluctuating disease activity that results in tissue damage and potentially life-threatening multiorgan failure. ${ }^{1}$ The incidence of SLE is variable but generally estimated to be about 1-10 per 100000 person-years with a prevalence of 20-70 per 100000 according to the different parts of the world. It is about 10 times more frequent in women. ${ }^{2-4}$

The complement system is an important effector pathway of innate immunity and plays a major role in SLE. ${ }^{5}$ Complement participation may occur in at least two ways. First, deficit of some components has been implicated as a risk factor for disease development. ${ }^{56}$ In particular, complete deficiency of classical pathway complement component C1 has been associated with an elevated risk of SLE in up to $90 \%$ of individuals with this condition. ${ }^{78}$ Other complement components such as C2 and C4 have also been associated. ${ }^{8}$ Second, the intense activation of complement has been linked to disease activity and damage to certain organs. ${ }^{56}$ Many complement products have been assigned different degrees of clinical value in assessing disease activity, such as serum CH50, levels of C3, C4, C3d, C4d, C3a, C4a, C5b-9, Ba, Bb and urine C3d. ${ }^{6} 10$

Our objective was to estimate the impact of complement level changes on clinical manifestations, visceral injury and mortality in our patients with SLE, with special interest in patients with continuous low complement levels compared with patients with fluctuating and/or continuous normal levels.

\section{METHODS}

We retrospectively included all patients with lupus who were seen at the Hospital Italiano de Buenos Aires from year 2000 to 2013. The inclusion criteria were fulfilment of American College of Rheumatology (ACR) or Systemic Lupus International Collaborating Clinics (SLICC) criteria, 18 years of age or older and at least two C3 and C4 determinations, measured by nephelometry, during 
follow-up. Low C3 was defined when serum levels were lower than $83 \mathrm{mg} / \mathrm{dL}$ and low $\mathrm{C} 4$ when serum levels were lower than $10 \mathrm{mg} / \mathrm{dL}$.

Patients were divided in three groups: those with persistent normal complement levels (all measurements above normal levels), those with persistent low complement levels (all measurements below normal levels) and finally those with fluctuant complement levels (at least one normal measurement and at least one low measurement).

The following outcomes were compared between the three groups: clinical features, damage at the end of follow-up (measured by SLICC/ACR damage index) and mortality.

Renal involvement was defined as renal failure and/ or microhematuria and/or proteinuria $(>500 \mathrm{mg} / 24 \mathrm{~h})$ plus biopsy that shows lupus patterns. Neurological involvement was defined as the presence of psychosis, seizures, myelitis, multiple mononeuritis, acute confusional syndrome and peripheral or cranial neuropathy that could not be explained by other reason. Leucopaenia, lymphopaenia and thrombocytopaenia were defined as cell counts $<4000 \mathrm{cell} / \mathrm{mm}^{3}<1000 \mathrm{cell} / \mathrm{mm}^{3}$ and $<100000 \mathrm{cell} / \mathrm{mm}^{3}$, respectively, that could not be explained by other means. Haemolytic anaemia was defined as anaemia with haemolytic parameters (rise of indirect bilirubin and lactate dehydrogenase, and/or haptoglobulin declines) plus positive Coombs test. Drugs (hydroxychloroquine, corticosteroid pulse, corticosteroid, immunosuppressive therapy (cyclophosphamide, mycophenolate, azathioprine) and biological therapy (rituximab and belimumab)) were defined as dichotomy variable (received/nor received) at any time during follow-up.

Statistical analysis was performed with the STATA program V.14.1. Continuous variables were computed as mean and SD or median with IQR, depending on the distribution of the variable. Categorical variables were presented as number and percentage. Analysis of variance test was used to compare continuous variables. $\chi^{2}$ and Fisher tests were used to compare categorical variables. A Cox proportional hazard model was used to estimate the association between complement level group and mortality or organ damage, adjusting by age, sex, disease duration, organs involvement, haematological involvement, drugs used and anti-dsDNA. A $p$ value of $<0.05$ was considered significant.

\section{RESULTS}

We included 270 patients, 242 (89.6\%) females with a mean age at diagnosis of 34.2 years (SD 15.8) (table 1). One hundred and sixteen patients $(43 \%)$ had persistently normal complement, 79 patients (29\%) had persistently low complement levels and 75 patients (28\%) had fluctuant levels. The median of complement determinations throughout the follow-up period was 7 (range:2-23) without differences among complement groups. Only $43(16 \%)$ patients were classified based on only two samples.

Table 1 shows cumulative clinical manifestations in the three groups. The only significant difference were

Table 1 Patients demographic, cumulative clinical data and autoantibodies profile by complement group

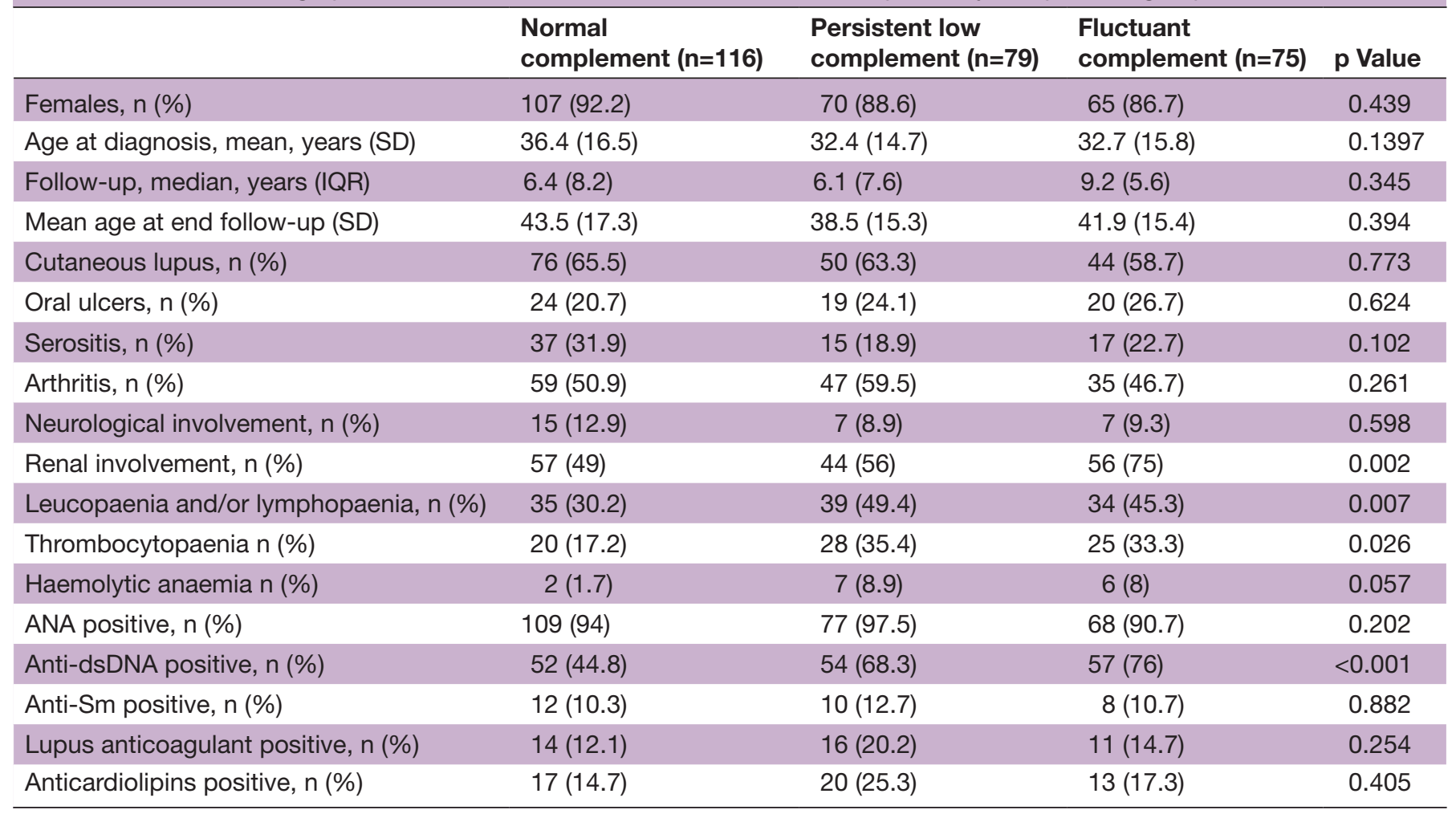


Table 2 Univariate and multiple Cox regression analysis with damage (SLICC/ACR $\geq 1$ ) as dependent variable, adjusting by patients' characteristics and treatment.

\begin{tabular}{lllllll}
\hline & \multicolumn{3}{l}{ Univariate Cox regression } & \multicolumn{3}{l}{ Multiple Cox regression } \\
\cline { 2 - 7 } & HR & $\mathbf{9 5 \%} \mathbf{C l}$ & $\mathbf{p}$ Value & HR & $\mathbf{9 5 \%} \mathbf{C l}$ & p Value \\
\hline Age at diagnosis & 1.01 & 0.99 to 1.02 & 0.052 & 1.01 & 1.006 to 1.03 & 0.002 \\
\hline Sex & 1.45 & 0.89 to 2.35 & 0.131 & 1.3 & 0.76 to 2.21 & 0.325 \\
Renal impairment & 1.06 & 0.76 to 1.49 & 0.695 & 1.08 & 0.73 to 1.59 & 0.685 \\
\hline Neurological impairment & 1.57 & 1.005 to 2.46 & 0.047 & 1.78 & 1.05 to 3.02 & 0.03 \\
\hline Leucopaenia or lymphopaenia & 1.003 & 0.72 to 1.39 & 0.983 & 0.92 & 0.65 to 1.31 & 0.651 \\
\hline Thrombocytopaenia & 1.46 & 1.03 to 2.07 & 0.031 & 1.79 & 1.21 to 2.63 & 0.003 \\
\hline Haemolytic anaemia & 1.09 & 0.55 to 2.15 & 0.794 & 0.97 & 0.47 to 2.02 & 0.954 \\
\hline Persistent low complement & 1.29 & 0.86 to 1.92 & 0.204 & 1.29 & 0.83 to 2.01 & 0.257 \\
\hline Fluctuant complement & 0.83 & 0.56 to 1.24 & 0.384 & 0.67 & 0.42 to 1.05 & 0.081 \\
\hline Hydroxychloroquine & 0.67 & 0.41 to 1.109 & 0.121 & 0.37 & 0.21 to 0.66 & 0.001 \\
\hline Corticosteroid pulse & 1.17 & 0.83 to 1.66 & 0.358 & 1.05 & 0.64 to 1.70 & 0.843 \\
Corticosteroid & 1.6 & 0.78 to 3.28 & 0.193 & 2.34 & 1.01 to 5.42 & 0.046 \\
\hline Immunosuppressive drugs (cyclophosphamide, & 1.5 & 1.07 to 2.105 & 0.018 & 1.57 & 0.99 to 2.48 & 0.052 \\
mycophenolate and azathioprine) & & & & & & \\
\hline Biological therapy (rituximab and belimumab) & 1.38 & 0.74 to 2.57 & 0.301 & 1.14 & 0.59 to 2.18 & 0.693 \\
\hline Anti-dsDNA & 1.12 & 0.8 to 1.57 & 0.5 & 1.05 & 0.74 to 1.50 & 0.758 \\
\hline
\end{tabular}

SLICC/ACR, Systemic Lupus International Collaborating Clinics/American College of Rheumatology Damage Index for Systemic Lupus Erythematosus.

renal impairment, more frequent in the fluctuant group, leucopaenia, lymphopaenia and thrombocytopaenia and anti-double stranded DNA antibodies (dsDNA), more frequent in the fluctuant and persistently low complement groups.

Regarding damage at the end of the follow-up, $53 \%$ of the patients had SLICC/ACR equal to or greater than one. When Cox proportional hazard models were used (table 2) age at diagnosis, neurological impairment, thrombocytopaenia and corticosteroids were associated with more damage (HR 1.01; 1.78; 1.79; 2.34, respectively). Hydroxychloroquine (HCQ) behaved as a protective variable (HR 0.37; IC 95\% 0.21-0.66; $\mathrm{p}<$ $0.001)$. None of the complement groups were associated with accumulated damage.

Fourteen $(6 \%)$ patients died. There were no significant differences on survival between the three complement groups. At 10 years survival was 93\%, $93.5 \%$ and $92 \%$ for the normal complement group, the persistently low group and the fluctuant group, respectively. Six patients died because of infections, three died because of lupus activity and five died from other causes (cancer, unknown). In the fluctuant group almost all causes of mortality were infections, whereas in the persistently low group, they were equally divided between infections and activity and in the normal group, mortality was associated with other causes. There were significant differences in mortality causes between the fluctuant and the normal groups $(p=0.008)$.
We analysed the different variables and their influence on mortality. We found that HCQ was a protective variable (HR $0.18 ; 95 \%$ CI 0.039 to $0.92 ; \mathrm{p}=0.040$ ), while thrombocytopaenia (HR 3.2; $95 \%$ CI 1.005 to 10.3 ; $\mathrm{p}=0.049$ ) and age at diagnosis (HR 1.07; 95\% CI 1.03 to $1.1 ; \mathrm{p}<0.001)$ were associated with increased mortality risk. The presence of anti-dsDNA was not associated with increased mortality (HR: $0.34 ; 95 \% 0.09$ to 1.23; $\mathrm{p}=0.100$ ). The different complement groups were not associated with mortality.

\section{DISCUSSION}

This paper compares the characteristics of lupus patients according to serum complement levels, to ascertain whether differences in these levels may explain different course or clinical characteristics. This approach may be unique in that we divided groups according to normal complement levels, persistently low complement levels and fluctuant complement levels.

There is recognition that complement levels and SLE are intimately associated, but there still is controversy regarding the value of serum C3 and serum C4 as biomarkers of lupus disease activity. ${ }^{9-19}$ Although we found, as in other reports, that decreased levels of serum C3 and C4 were observed in patients with renal and haematological disease, ${ }^{20}$ our work revealed that behaviour of complement levels is heterogeneous in SLE. 
Damage at the end of follow-up occurred in $53 \%$ of the patients, and we did not find differences between complements groups. This is similar with the findings of Petri et $a l,{ }^{21}$ who did not find an association between complement consumption and damage. The strongest predictors of damage in their study appeared to be age and current corticosteroid dose.

Like in other series, the 10-year survival rate was nearly $90 \%,{ }^{22}$ and according to our results, complement behaviour did not seem to influence survival. It was interesting, however, to note that in the fluctuant complement group almost all deaths were due to infections, in the persistently low group they were equally distributed between infections and disease activity and in the normal group they were all from other causes neither related to infections nor to lupus activity. These findings may suggest that patients with fluctuant and persistently low level complement had more active disease and might have received more immunosuppressive therapy.

We found that HCQ was a protective factor both for mortality and damage, similar to other series. ${ }^{23-26}$ However, thrombocytopaenia was a mortality risk factor, in agreement with two large and long-term studies. ${ }^{27} 28$ Other studies, however, have shown no influence of thrombocytopaenia on survival. ${ }^{29}{ }^{30}$ Thrombocytopaenia may therefore be debatably regarded as a prognostic indicator of reduced survival. ${ }^{31}$ As in other studies older age at diagnosis was a death risk factor. ${ }^{32} 33$

A limitation of this study is that we did not correlate disease activity with complement levels. Another limitation is that in the persistently low complement group, we did not divide the patients into those who only had low $\mathrm{C} 3$ or low $\mathrm{C} 4$, so low levels could have been due to congenital complement deficit. However, the proportion of patients with low complement values due to deficit and not to disease activity is low in the literature. ${ }^{8}$ Related to drugs use, we did not determinate doses and periods of times because records were incomplete for these data.

In summary, our data suggest that patients with persistently normal levels of complement have lower prevalence of leucopaenia, lymphopaenia, thrombocytopaenia and antibodies to dsDNA. However, patients with fluctuant complement levels have higher renal impairment. Persistently low complement or fluctuant complement level did not increase visceral damage and/ or mortality in these patients.

Contributors All authors contributed to the conception and design of the study, acquisition and/or interpretation of data, drafting the article or revising it critically for important intellectual content and approved the final version of the manuscript.

Competing interests None declared.

Provenance and peer review Not commissioned; externally peer reviewed.

Open Access This is an Open Access article distributed in accordance with the Creative Commons Attribution Non Commercial (CC BY-NC 4.0) license, which permits others to distribute, remix, adapt, build upon this work non-commercially, and license their derivative works on different terms, provided the original work is properly cited and the use is non-commercial. See: http://creativecommons.org/ licenses/by-nc/4.0/ (c) Article author(s) (or their employer(s) unless otherwise stated in the text of the article) 2017. All rights reserved. No commercial use is permitted unless otherwise expressly granted.

\section{REFERENCES}

1. Rahman A, Isenberg DA. Systemic lupus erythematosus. N Engl J Med 2008;358:929-39.

2. Pons-Estel GJ, Alarcón GS, Scofield L, et al. Understanding the epidemiology and progression of systemic lupus erythematosus. Semin Arthritis Rheum 2010;39:257-68.

3. Scolnik M, Marin J, Valeiras SM, et al. Incidence and prevalence of lupus in Buenos Aires, Argentina: a 11-year health management organisation-based study. Lupus Sci Med 2014;1:e000021.

4. Ahmadpoor P, Dalili N, Rostami M. An update on pathogenesis of systemic lupus erythematosus. Iran J Kidney Dis 2014;8:171-84.

5. Manderson AP, Botto M, Walport MJ. The role of complement in the development of systemic lupus erythematosus. Annu Rev Immunol 2004;22:431-56.

6. Tsokos GC. Exploring complement activation to develop biomarkers for systemic lupus erythematosus. Arthritis Rheum 2004;50:3404-7.

7. Bowness P, Davies KA, Norsworthy PJ, et al. Hereditary C1q deficiency and systemic lupus erythematosus. QJM 1994;87:455-64

8. Holers VM, states Cdeficiency. Disease susceptibility, and infection risk in systemic lupus erythematosus. Arthritis Rheum 1999;42:2023-5.

9. Manzi S, Rairie JE, Carpenter AB, et al. Sensitivity and specificity of plasma and urine complement split products as indicators of lupus disease activity. Arthritis Rheum 1996;39:1178-88.

10. Lloyd W, Schur PH. Immune complexes, complement, and anti-DNA in exacerbations of systemic lupus erythematosus (SLE). Medicine 1981;60:208-17.

11. Hopkins P, Belmont HM, Buyon J, et al. Increased levels of plasma anaphylatoxins in systemic lupus erythematosus predict flares of the disease and may elicit vascular injury in lupus cerebritis. Arthritis Rheum 1988;31:632-41.

12. Sturfelt G, Johnson U, Sjöholm AG. Sequential studies of complement activation in systemic lupus erythematosus. Scand $J$ Rheumatol 1985;14:184-96.

13. Buyon JP, Tamerius J, Belmont HM, et al. Assessment of disease activity and impending flare in patients with systemic lupus erythematosus. comparison of the use of complement split products and conventional measurements of complement. Arthritis Rheum 1992;35:1028-37.

14. Falk RJ, Dalmasso AP, Kim Y, et al. Radioimmunoassay of the attack complex of complement in serum from patients with systemic lupus erythematosus. N Engl J Med 1985;312:1594-9.

15. Mollnes TE, Haga HJ, Brun JG, et al. Complement activation in patients with systemic lupus erythematosus without nephritis. Rheumatology 1999;38:933-40.

16. Abrass CK, Nies KM, Louie JS, et al. Correlation and predictive accuracy of circulating immune complexes with disease activity in patients with systemic lupus erythematosus. Arthritis Rheum 1980;23:273-82.

17. Esdaile JM, Joseph L, Abrahamowicz M, et al. Routine immunologic tests in systemic lupus erythematosus: is there a need for more studies? J Rheumatol 1996;23:1891-6.

18. Esdaile JM, Abrahamowicz M, Joseph L, et al. Laboratory tests as predictors of disease exacerbations in systemic lupus erythematosus. why some tests fail. Arthritis Rheum 1996;39:370-8.

19. Kao AH, Navratil JS, Ruffing MJ, et al. Erythrocyte C3d and C4d for monitoring disease activity in systemic lupus erythematosus. Arthritis Rheum 2010:62:837-44.

20. Ho A, Barr SG, Magder LS, et al. A decrease in complement is associated with increased renal and hematologic activity in patients with systemic lupus erythematosus. Arthritis Rheum 2001;44:2350-7.

21. Petri M, Purvey S, Fang H, et al. Predictors of organ damage in systemic lupus erythematosus: the Hopkins Lupus Cohort. Arthritis Rheum 2012;64:4021-8.

22. Kasitanon N, Magder LS, Petri M. Predictors of survival in systemic lupus erythematosus. Medicine 2006;85:147-56.

23. Akhavan PS, Su J, Lou W, et al. The early protective effect of hydroxychloroquine on the risk of cumulative damage in patients with systemic lupus erythematosus. J Rheumatol 2013;40:831-41.

24. Ruiz-Irastorza G, Egurbide MV, Pijoan Jl, et al. Effect of antimalarials on thrombosis and survival in patients with systemic lupus erythematosus. Lupus 2006;15:577-83.

25. Alarcón GS, McGwin G, Bertoli AM, et al. Effect of hydroxychloroquine on the survival of patients with systemic lupus 
erythematosus: data from LUMINA, a multiethnic US cohort (LUMINA L). Ann Rheum Dis 2007;66:1168-72.

26. Shinjo SK, Bonfá E, Wojdyla D, et al. Antimalarial treatment may have a time-dependent effect on lupus survival: data from a multinational latin american inception cohort. Arthritis Rheum 2010;62:855-62.

27. Reveille JD, Bartolucci A, Alarcón GS. Prognosis in systemic lupus erythematosus. negative impact of increasing age at onset, black race, and thrombocytopenia, as well as causes of death. Arthritis Rheum 1990;33:37-48.

28. Pistiner M, Wallace DJ, Nessim S, et al. Lupus erythematosus in the 1980s: a survey of 570 patients. Semin Arthritis Rheum 1991;21:55-64.

29. Mestanza-Peralta M, Ariza-Ariza R, Cardiel MH, et al. Thrombocytopenic Purpura as initial manifestation of systemic lupus erythematosus. J Rheumatol 1997;24:867-70.

30. Swaak AJ, Nossent JC, Bronsveld W, et al. Systemic lupus erythematosus. I. Outcome and survival: dutch experience with 110 patients studied prospectively. Ann Rheum Dis 1989;48:447-54.

31. Fayyaz A, Igoe A, Kurien BT, et al. Haematological manifestations of lupus. Lupus Sci Med 2015;2:e000078.

32. Merola JF, Bermas B, Lu B, et al. Clinical manifestations and survival among adults with (SLE) according to age at diagnosis. Lupus 2014;23:778-84.

33. Lin H, Wei JC, Tan CY, et al. Survival analysis of late-onset systemic lupus erythematosus: a cohort study in China. Clin Rheumatol 2012;31:1683-9. 